\title{
VESICULAR GLUTAMATE TRANSPORTER 1 IMMUNOSTAINING IN THE NORMAL AND EPILEPTIC HUMAN CEREBRAL CORTEX
}

\author{
L. ALONSO-NANCLARES AND J. DeFELIPE* \\ Instituto Cajal (CSIC), Ave. Dr. Arce 37, 28002 Madrid, Spain
}

\begin{abstract}
Glutamate is the main excitatory neurotransmitter in the brain where, due to the activity of specific vesicular glutamate transporters, it accumulates in synaptic vesicles. The vesicular glutamate transporter 1 is found in the majority of axon terminals that form asymmetrical (excitatory) synapses in the rat neocortex. However, since there is no information available regarding the distribution of vesicular glutamate transporter 1 in the human neocortex, we have used correlative light and electron microscopy to define its expression in this tissue. We found that the distribution of vesicular glutamate transporter 1-immunoreactivity is virtually identical to that found in the rat neocortex, both at the light and electron microscope levels. Therefore, we assessed whether vesicular glutamate transporter 1 immunostaining might be a useful tool to study the pathological alterations of glutamatergic transmission in the epileptic cerebral cortex. We analyzed the distribution of vesicular glutamate transporter 1 in the peritumoral neocortex of patients with epilepsy secondary to low-grade tumors. In these regions, we found alterations in the pattern of vesicular glutamate transporter 1-immunoreactivity that perfectly matched the neuronal loss and gliosis, as well as the decrease in the number of asymmetrical synapses identified by electron microscopy in this tissue. Thus, vesicular glutamate transporter 1 immunostaining appears to be a reliable and simple tool to study glutamatergic synapses in the normal and epileptic human cerebral cortex. @ 2005 IBRO. Published by Elsevier Ltd. All rights reserved.
\end{abstract}

Key words: electron microscopy, epilepsy, excitatory synapses, peritumoral cortex, synaptic circuits, tumors.

Pyramidal cells generate the vast majority of excitatory synapses in the cerebral cortex, which are formed by glutamatergic axon terminals that establish asymmetrical contacts (Peters, 1987). These asymmetrical synapses are principally distinguished by their prominent postsynaptic density, and this morphological criterion has been virtually the only reliable tool to identify putative excitatory glutamatergic synapses (Gray, 1959; Colonnier, 1968, 1981; Peters, 1987; Peters et al., 1991; Peters and Palay, 1996). As a result, indirect electron microscopy studies have been the only means to analyze excitatory synaptic circuits, thereby hindering the localization of glutamatergic

*Corresponding author. Tel: +34-91-585-47-34; fax: +34-91-585-47-54. E-mail address: defelipe@cajal.csic.es (J. DeFelipe).

Abbreviations: ECoG, electrocorticography; -ir, immunoreactivity/ immunoreactive; NeuN, neuron-specific nuclear protein; PB, phosphate buffer; VGLUTs, vesicular glutamate transporters; VGLUT1, VGLUT2 and VGLUT3, vesicular glutamate transporters 1, 2 and 3. terminals and impairing the study of the possible diseaserelated alterations in these excitatory circuits.

It has recently been demonstrated that immunostaining for the vesicular glutamate transporters (VGLUTs) is a reliable tool to examine the glutamatergic system in the brain. It is know that glutamate accumulates in synaptic vesicles due to the activity of specific vesicular proteins, the VGLUTs. To date, three VGLUTs have been identified in the rat CNS, differentially distributed in various brain regions: VGLUT1 (Bellocchio et al., 2000; Takamori et al., 2000), VGLUT2 (Aihara et al., 2000; Fremeau et al., 2001; Varoqui et al., 2002) and VGLUT3 (Fremeau et al., 2002; Gras et al., 2002). VGLUT1 is expressed in a subset of excitatory neurons mainly in telencephalic regions such as the neocortex, hippocampal formation, amygdaloid nuclei, anterior olfactory nucleus and olfactory bulb ( $\mathrm{Ni}$ et al., 1994, 1995, 1996; Bellocchio et al., 1998; Fujiyama et al., 2001; Fremeau et al., 2001; Herzog et al., 2001; Varoqui et al., 2002; Kaneko et al., 2002; reviewed in Kaneko and Fujiyama, 2002). Furthermore, VGLUT1 has been shown to be the most abundant VGLUT in the neocortex. Ultrastructural studies have also shown that in the neocortex, the synaptic vesicles that accumulate VGLUT1 are exclusively found in axon terminals that form asymmetrical synapses (Bellocchio et al., 1998; Fujiyama et al., 2001; Fremeau et al., 2001; Kaneko et al., 2002; AlonsoNanclares et al., 2004).

The identification of specific markers for glutamatergic synapses is particularly relevant to study the pathological alterations in the cerebral cortex in which glutamatergic transmission is thought to play a critical role. Epilepsy is one condition in which such alterations are important and that is frequently associated with a variety of brain lesions, tumors or other structural abnormalities that affect the cerebral cortex. Despite the years spent in studying this phenomenon, identifying the cellular mechanisms that provoke seizures is still one of the main goals of research into epilepsy. It has been shown that both glutamate and GABA are involved in the initiation and the spreading of seizure activity. Moreover, alterations in both these systems of transmission have been related to the onset and development of epilepsy (Ben Ari and Represa, 1990; Sloviter, 1991; Avanzini et al., 1992; Schwartzkroin, 1994; McNamara, 1994; Chapman, 1998 Bernard et al., 2000; Demarque et al., 2004). However, the analysis of the anatomical substrate of epilepsy in the neocortex has generally focused on the morphological alterations in GABAergic inhibitory circuits (e.g. Ribak et al.,1989; Houser et al., 1986; Avanzini et al., 1992; Spreafico et al., 1993; DeFelipe, 1999; Silva et al., 2002). Apart from conventional

0306-4522/05 $\$ 30.00+0.00 \odot 2005$ IBRO. Published by Elsevier Ltd. All rights reserved. doi:10.1016/j.neuroscience.2005.03.038 
electron microscopy with its associated drawbacks, there is a notable lack of reliable tools to study glutamatergic systems. Furthermore, the vast majority of studies of the expression VGLUTs in the cerebral cortex have been performed on rats and few studies have focused on the human neocortex (Ni et al., 1996; Takamori et al., 2000).

Thus, one of the main goals of the present study was to examine the distribution and connectivity of VGLUT1immunoreactive (-ir) profiles in the normal human neocortex. We also set out to assess whether this marker could be used to examine alterations of glutamatergic neuronal circuits in the human epileptic cortex. For this purpose, we studied the peritumoral neocortex of patients with epilepsy that was secondary to low-grade tumors. This is a common cause of epilepsy in which the epileptogenic region presents a loss of neurons and excitatory synapses (Cascino, 1990; Plate et al., 1993; Marco et al., 1997; Blümcke et al., 1999). As a result, we confirm that VGLUT1 is a suitable marker to study glutamatergic synapses in the normal and epileptic human cerebral cortex, demonstrating that certain glutamatergic circuits are affected in the peritumoral epileptic neocortex.

\section{EXPERIMENTAL PROCEDURES}

Human brain tissue was obtained from subjects that had undergone an anterior temporal lobectomy at the Neurosurgery Department of the "La Princesa" Hospital (Madrid, Spain). Surgery was performed only after the patient or a parent had given informed consent, according to the Helsinki Declaration (1991). Both the institutional ethical committee of the hospital and of our institution, approved the surgical protocols and those used in the manipulation of the human tissue. We selected patients with hippocampal sclerosis or with brain tumors. In both cases, a tailored temporal lobectomy was performed using electrocorticography (ECoG) guidance in all cases. Briefly, ECoG involved, placing a $4 \times 5$ grid of $1.2 \mathrm{~mm}$ diameter electrodes (Pt/lr) embedded in Sylastic with an inter-electrode distance of $1 \mathrm{~cm}$ center-to-center (Add-Tech $\AA$, Medical Instrument Cooperation, Racine, WI, USA), directly over the exposed temporal lateral cortex. Spiking areas were identified from the electrodes exhibiting spikes ( $<80 \mathrm{~ms}$ ) or sharp waves (80-200 ms) (Ajmone-Marsan and O'Connor, 1973; Chatrian and Quesney, 1999) at a mean frequency greater than $1 \mathrm{spike} / \mathrm{min}$. Non-spiking areas were defined by electrodes where no spikes, sharp waves or slow activity was observed. The outcome was evaluated using Engel's scale (Engel, 1987) at least 1 year after surgery. Control tissue consisted of non-spiking temporal neocortex with no histopathological abnormalities, obtained from three epileptic patients with hippocampal sclerosis (three male individuals: $\mathrm{H} 200, \mathrm{H} 212$ and $\mathrm{H} 213$ of 27,24 and 38 years old, respec- tively). The peritumoral tissue was obtained following the resection of the epileptogenic temporal cortex from seven epileptic patients with brain tumors. The tumors were classified according to the World Health Organization (WHO) histological typing of tumors in the CNS (Kleihues and Cavenee, 2000). The cases studied including six glioneuronal hamartomatous tumors and one ganglioglioma as specified, along with further clinical details, in Table 1.

Upon removal, the resected brain tissue was immediately fixed in cold $4 \%$ paraformaldehyde in phosphate buffer $0.1 \mathrm{M}, \mathrm{pH}$ 7.4 (PB). After $2 \mathrm{~h}$, the tissue was cut into small $\sim 1 \mathrm{~cm}^{3}$ blocks that were postfixed in the same fixative for $24-48 \mathrm{~h}$ at $4{ }^{\circ} \mathrm{C}$. Coronal $100 \mu \mathrm{m}$ thick sections were cut with a Vibratome and collected serially in PB. Some sections were stained with Toluidine Blue to reveal the laminar boundaries and to study the cytoarchitectonic alterations.

\section{Immunocytochemistry}

Free-floating sections were initially pretreated in $1 \% \mathrm{H}_{2} \mathrm{O}_{2}$ for 30 $\mathrm{min}$ to remove endogenous peroxidase activity, and then for $1 \mathrm{~h}$ in PB with $0.25 \%$ Triton X-100 and 3\% normal goat or horse serum (for polyclonal antisera and monoclonal antibodies respectively; Vector Laboratories Inc., Burlingame, CA, USA). The sections were then incubated overnight at $4{ }^{\circ} \mathrm{C}$ with a guinea-pig antiVGLUT1 antibody (1:5000; AB5905, Chemicon International, Temecula, CA, USA), a mouse antiserum raised against the neuronspecific nuclear protein (NeuN, 1:2000; Chemicon) and a mouse anti-GFAP monoclonal antibody (1:4000; Sigma, St. Louis, MI, USA). The following day, the sections were rinsed and incubated for $2 \mathrm{~h}$ using goat anti-guinea-pig (VGLUT1 immunostaining) or horse anti-mouse (NeuN and GFAP immunostaining) biotinylated antibodies (1:200; BA-1000; Vector). Sections were subsequently incubated for $1 \mathrm{~h}$ in an avidin-biotin peroxidase complex (Vectastain ABC Elite PK6100, Vector), and finally with the chromogen 3,3' diaminobenzidine tetrahydrochloride (DAB; Sigma-Aldrich, St. Louis, MO, USA). After staining the sections were dehydrated, cleared with xylene and coverslipped. Control sections were processed as above but without the primary antibody and no significant staining was seen under these conditions.

To generate the figures, images were captured with a digital camera (Olympus DP50) attached to an Olympus light microscope, and Adobe Photoshop 6.0 software (Adobe Systems, San Jose, CA, USA) was used to produce figure plates.

\section{Electron microscopy}

For electron microscopy, we followed the same protocol as laid out above except that Triton X-100 was excluded from the buffers. Once the immunocytochemical staining was complete, the sections were treated with $1 \%$ osmium tetroxide and flat embedded in Araldite resin. Plastic-embedded sections were studied by correlative light and electron microscopy as described elsewhere in more detail (DeFelipe and Fairén, 1993). Briefly, sections were

Table 1. Summary of clinical and surgical data

\begin{tabular}{|c|c|c|c|c|}
\hline Patient & $\begin{array}{l}\text { Age at the time of } \\
\text { surgery (years), sex, side }\end{array}$ & $\begin{array}{l}\text { Age at onset, duration } \\
\text { of seizures (years) }\end{array}$ & Tumoral lesion & $\begin{array}{l}\text { Engel scale for } \\
\text { surgical outcome }\end{array}$ \\
\hline H51 & $31, F, R$ & 25,6 & Hamartomatous & 1 \\
\hline H66 & $26, M, L$ & 9,17 & Hamartomatous & 1 \\
\hline $\mathrm{H} 73$ & $16, M, L$ & 15,1 & Hamartomatous & 1 \\
\hline $\mathrm{H} 163$ & $12, \mathrm{M}, \mathrm{R}$ & 11,1 & Hamartomatous & 1 \\
\hline H174 & $38, F, R$ & 8,30 & Ganglioglioma & 1 \\
\hline $\mathrm{H} 182$ & $23, F, R$ & 11,12 & Hamartomatous & 1 \\
\hline $\mathrm{H} 214$ & $33, M, L$ & 18,15 & Hamartomatous & 1 \\
\hline
\end{tabular}

Engel's scale (Engel, 1987); F, female; L, left hemisphere; M, male; R, right hemisphere. 
photographed under the light microscope and then they were cut again into serial semithin (2- $\mu \mathrm{m}$-thick) sections with a Reichert ultramicrotome. The semithin sections were examined under the light microscope and re-photographed. Selected semithin sections were resectioned into serial ultrathin sections with a silver-gray interference color that corresponded to a thickness of approximately 60-70 nm (Peachey, 1958). The ultrathin sections were collected on formvar-coated single-slot grids, stained with uranyl acetate and lead citrate, and examined with a JEOL 1200 EX electron microscope (JEOL USA, Inc., Peabody, MA, USA). Photographs were taken with a digitalizing image system (Mega View III Side-mounted TEM Camera, Soft Imaging System GmbH, Germany, 2003) using the analiSIS imaging acquisition software (analiSIS $® 3.2$, Soft Imaging system $\mathrm{GmbH}, 2003$ ).

\section{RESULTS}

\section{Light microscopy}

Normal neocortex. In accordance with data obtained from the rat neocortex (Bellocchio et al., 1998; Fremeau et al., 2001; Herzog et al., 2001; Kaneko and Fujiyama, 2002;
Kaneko et al., 2002; Alonso-Nanclares et al., 2004), VGLUT1-ir was homogeneously distributed throughout the depth of the human neocortex (Fig. 1). In all layers, VGLUT1-ir was associated with punctate structures of diverse sizes, all of which appeared to be less than approximately $2 \mu \mathrm{m}$. No ir was detected in cell bodies. These VGLUT1-ir punctate structures were numerous and widely dispersed in the neuropil without any obvious pattern in their distribution (Fig. 1C). Furthermore, VGLUT1-ir could be seen to outline the somata and proximal dendritic segments of neurons, many of which could be classified as pyramidal cells due to their shape and prominent apical dendrite (Fig. 1D).

Peritumoral neocortex. The peritumoral cortex was defined as the macroscopically normal cortical region adjacent to the tumor mass. However, in Toluidine Bluestained sections the histopathological abnormalities were readily apparent in these regions (Figs. 2, 3). These alter-

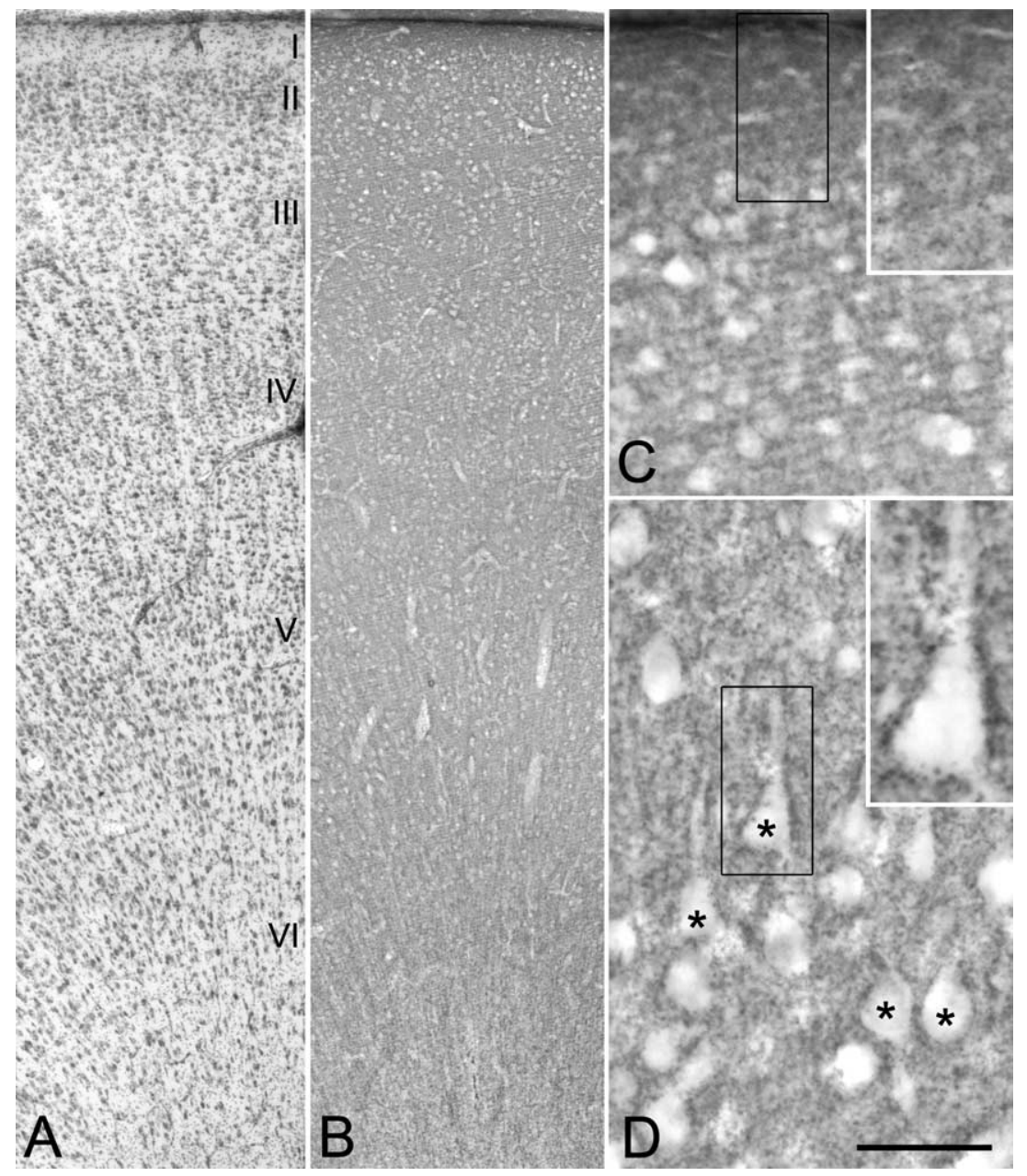

Fig. 1. Normal human temporal neocortex. (A) Low-power photomicrograph of a $100 \mu \mathrm{m}$-thick vibratome section stained with Toluidine Blue to identify the cortical layers. (B) Adjacent section immunostained for VGLUT1, showing the laminar pattern of the ir profiles. (C) Higher magnification of layer I to layer II, to illustrate the rather homogeneous distribution of ir puncta. The inset is a higher magnification of the boxed area to show VGLUT1-ir punctate profiles in layer I. (D) Higher magnification from layer III to illustrate unlabeled pyramidal cell somata (some indicated by asterisks), surrounded by VGLUT1-ir punctate profiles. The inset shows a higher magnification the boxed area to illustrate a pyramidal cell with its apical dendrite outlined by VGLUT1 profiles. Scale bar $=460 \mu \mathrm{m}$ (shown in D) in A and B, $60 \mu \mathrm{m}$ in C and D, and $30 \mu \mathrm{m}$ in the insets in C and D. 

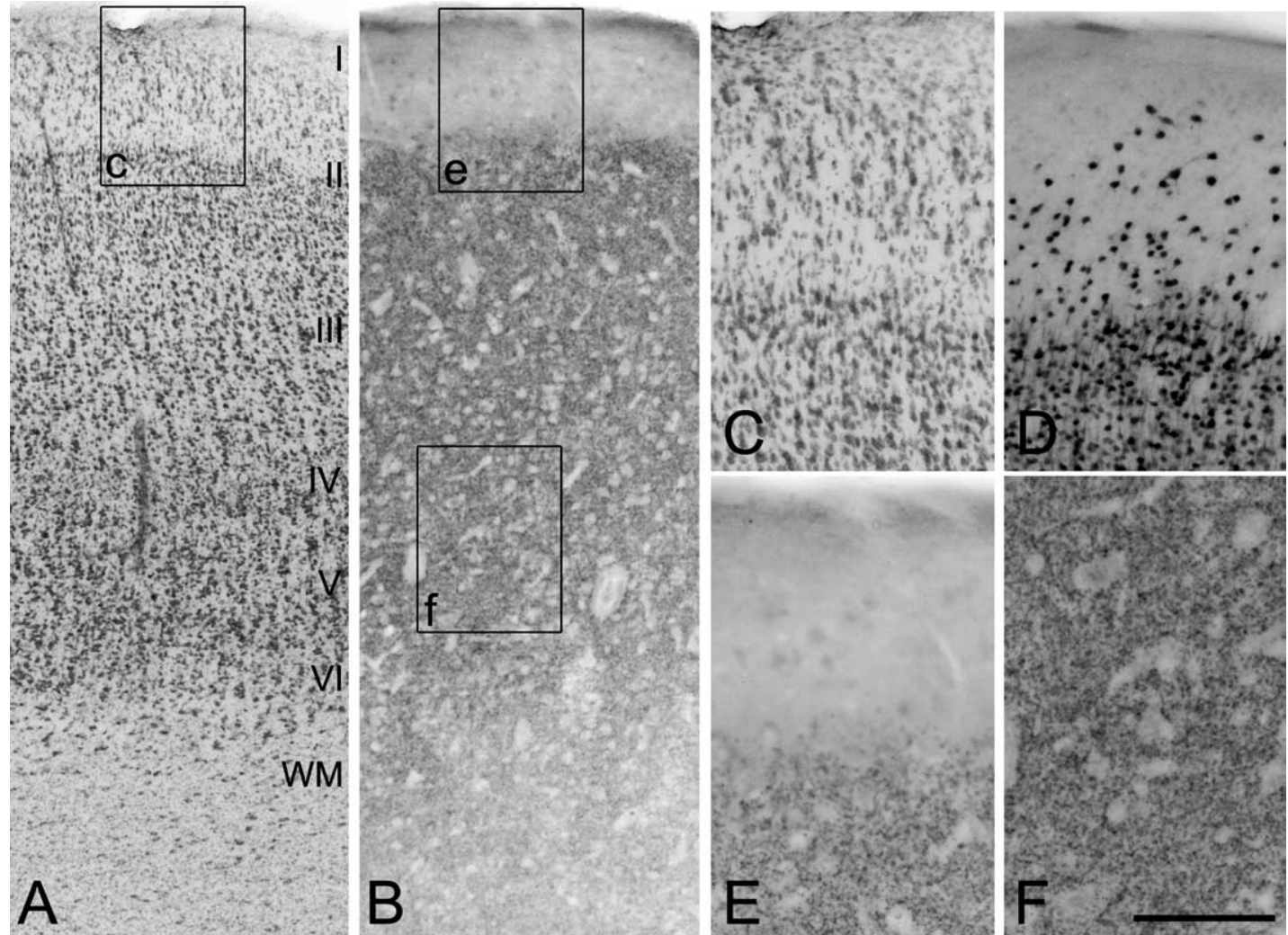

Fig. 2. Peritumoral neocortex from patient $\mathrm{H} 66$ to illustrate the alterations in layers I and II. (A, B) Low-power photomicrographs of $100 \mu \mathrm{m}$-thick vibratome sections stained with Toluidine Blue (A) and VGLUT1 (B). (C) Higher magnification of the boxed region in A, to illustrate gliosis with alterations in the cytoarchitecture of layers I-II. (D) Photomicrograph to illustrate the same region as in C, in an adjacent NeuN-immunostained section showing the presence of relatively large numbers of NeuN-ir neurons. (E, F) Photomicrographs at higher magnification of the boxed regions e and $f$ in B. In E, layers I and II are shown to highlight the lack of VGLUT1-ir axon terminals, while in the deeper layers of the same section there are numerous ir boutons ( $F$ ). Scale bar $=475 \mu \mathrm{m}$ (shown in $F$ ) in A and B, and $235 \mu \mathrm{m}$ in $\mathrm{C}-\mathrm{F}$.

ations included an intense gliosis, uncharacteristic cell proliferation and neuronal loss, all of which were contiguous with other normal looking regions. This loss of neurons was particularly evident in sections stained for $\mathrm{NeuN}$, an antibody that specifically recognizes a soluble, nuclear, neural vertebrate DNA-binding protein. NeuN is present in the nucleus and perikarya, including proximal dendritic processes, of the vast majority of mature neurons in both the central and peripheral nervous systems of different vertebrate species, including humans (Mullen et al., 1992; Wolf et al., 1996; Sarnat et al., 1998).

When adjacent sections to those showing neuronal loss and gliosis were stained for VGLUT1, alterations in the pattern of VGLUT1-ir were evident. These changes consisted mainly of a severe decrease of VGLUT1-ir punctate structures. Moreover, these changes were heterogeneous within and between different patients. For instance, in some peritumoral regions there was a dramatic decrease of VGLUT1-ir in layers I to III, whereas in other regions of the same patient the decrease affected only layers III to V, layers $\mathrm{V}$ to $\mathrm{VI}$, or could be observed in all cortical layers. Nevertheless, the predominant alteration in the staining pattern was consistent in each patient. For example, the peritumoral region of the patient H66 (Fig. 2) was characterized by a dramatic decrease in VGLUT1-ir in layers I and II (Fig. 2E), while the other cortical layers remained unaffected (Fig. 2F). In contrast, the alterations in patient $\mathrm{H} 214$ were characterized by reduced labeling in the deeper regions of the neocortex, particularly in layers $\mathrm{V}$ to VI (Figs. 3, 4). Thus, the alterations in the pattern of VGLUT1-ir were variable in the peritumoral regions.

\section{Electron microscopy}

The two major morphological types of cortical synapses, denominated type I and type II by Gray (1959), or asymmetrical and symmetrical types according to Colonnier (1968; for review see Colonnier 1981; Peters, 1987; Peters et al., 1991; Peters and Palay, 1996; White, 1989), were clearly identified in the cortical tissue analyzed. The synapses in which the synaptic cleft and associated membrane densities could not be visualized clearly (due to the oblique plane of section) were also considered as uncharacterized synapses.

\section{Normal neocortex}

The VGLUT1-ir positive puncta identified throughout the neuropil of the temporal neocortex, from layer I to layer VI, were examined by electron microscopy. The labeling was located exclusively in axon terminals, which were filled with 

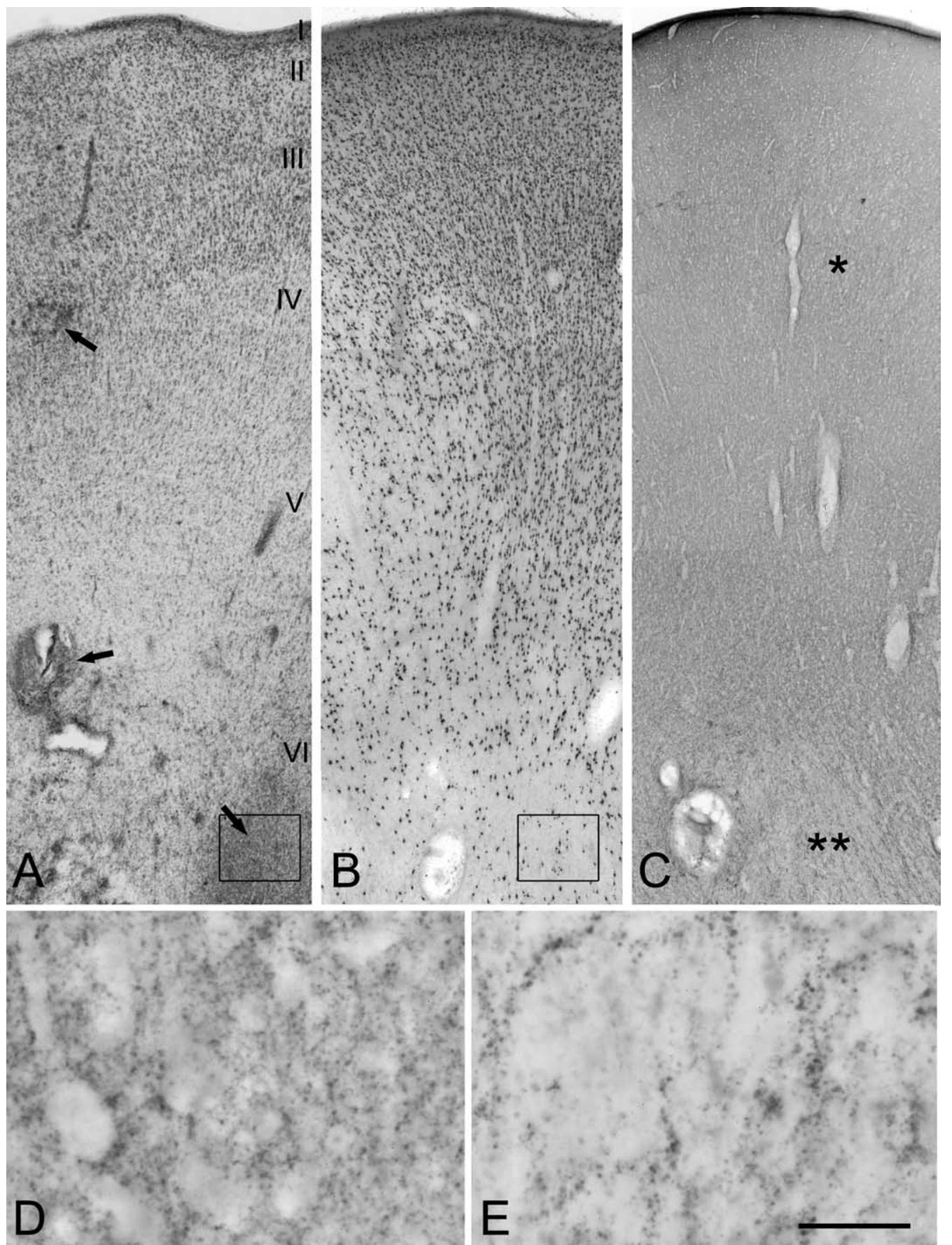

Fig. 3. Peritumoral neocortex from patient $\mathrm{H} 214$ to illustrate the alterations in the deeper layers. (A-C) Low power photomicrographs stained with Toluidine Blue (A), NeuN (B) and VGLUT1 (C). The arrows in A indicate cell masses composed of glial cells and uncharacterized small cells that in adjacent sections, correspond to areas with few neurons (in B stained for NeuN) and little VGLUT1 ir (indicated by two asterisks in C). (D, E) Higher magnification of the areas indicated by one and two asterisks, respectively. In D, the pattern of VGLUT1-ir is normal with numerous positive profiles. In E, a clear decrease in the number of VGLUT1-ir puncta can be seen. The boxed areas in A and B are shown at higher magnification in Fig. 4. Scale bar $=860 \mu \mathrm{m}$ (shown in $\mathrm{E}$ ): in $\mathrm{A}-\mathrm{C}$, and $40 \mu \mathrm{m}$ in $\mathrm{D}$ and $\mathrm{E}$.

immunostained vesicles (Fig. 5). As previously reported in the rat neocortex (Bellocchio et al., 1998; Fremeau et al., 2001; Fujiyama et al., 2001; Herzog et al., 2001; Kaneko et al., 2002; Alonso-Nanclares et al., 2004), the majority of these VGLUT1-ir axon terminals formed synaptic contacts with unstained dendritic shafts and spines. Furthermore, all the labeled synapses that could be morphologically identified were asymmetrical synapses ( $n=148$; Fig. 5B, C). As also occurs in the rat neocortex (Alonso-Nanclares et al., 2004), numerous VGLUT1-ir axon terminals were in apposition to the membrane of unlabeled neuronal cell bodies and proximal dendrites (Fig. 5D, E). 

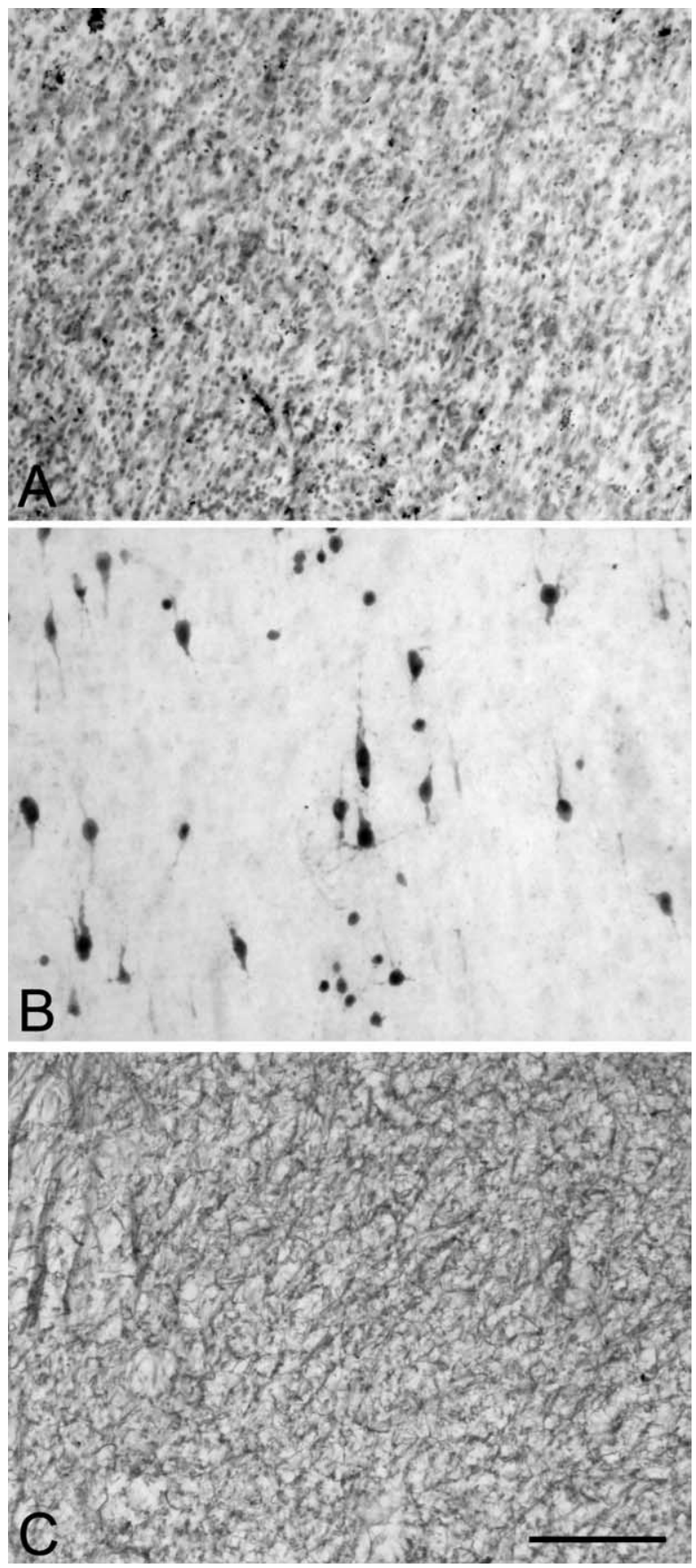

Fig. 4. (A, B) Photomicrographs at high magnification of the boxed areas shown in panels $A$ and $B$ of Fig. 3 . The tissue is stained with Toluidine Blue (A) and NeuN (B). In A, the cell mass shown is composed of glial cells, neurons and uncharacterized small cells. In the adjacent section stained for NeuN (B) relatively few neurons can be identified. (C) Photomicrograph illustrating intense GFAP immunostaining in a region adjacent to that shown in B. Scale bar $=130 \mu \mathrm{m}$ (shown in $\mathrm{C}$ ) in A-C.

Since pyramidal cell somata exclusively receive symmetrical synapses (White, 1989; DeFelipe and Fariñas, 1992), we examined whether the perisomatic VGLUT1-ir axon terminals around pyramidal cells formed symmetrical axosomatic synapses, or whether as in the rat neocortex
(Alonso-Nanclares et al., 2004), they established asymmetrical synapses with adjacent dendritic elements. We found that these VGLUT1-ir perisomatic axon terminals formed asymmetrical synapses with adjacent dendritic shafts and spines, but never with the cell body of the pyramidal neuron with which they were contiguous (Fig. $5 \mathrm{E})$. Therefore, the pattern of VGLUT1-ir appeared to be identical in the human to that of the rat at both light and electron microscope level.

\section{Peritumoral neocortex}

In order to examine whether the alterations in the pattern of ir observed at the light microscope level corresponded to alterations in the cortical circuits, we used correlative light and electron microscopy. There was a remarkable coincidence between the decrease in the density of the VGLUT1-ir profiles observed at the light microscope level and the decrease in the number of asymmetrical synapses accompanied by gliosis (Fig. 6). There was no evidence that the decrease in immunostaining was due to a reduction in the protein levels. Even within regions showing severe gliosis, the relatively few VGLUT1-ir axon terminals had a normal looking appearance and no unlabeled axon terminals were observed adjacent to the labeled ones (Fig. $6 \mathrm{~B})$. In general, the decrease of VGLUT1-ir puncta was associated with neuronal loss, as revealed in adjacent sections stained for Nissl or NeuN. Nevertheless, some regions that contained a relatively large number of neurons surprisingly showed a dramatic decrease of VGLUT1-ir. This was apparent in patient $\mathrm{H} 66$, who showed a dramatic decrease in the number of VGLUT1-ir puncta in layers I and II, despite the relatively large number of neurons that could be observed (Fig. 2). The ultrastructural examination of these layers in adjacent sections, revealed a lack of synapses (Fig. 6A), indicating that neurons within this region were not synaptically connected. However, in the rest of the cortical layers where gliosis was observed, numerous VGLUT1-ir axon terminals did form synapses (Fig. $6 B$ ). Similarly, in the case of patient $\mathrm{H} 214$, there was also a remarkable decrease in the number of synapses in regions well populated by neurons (panel e in Fig. 3; Fig. $6 D$ ), whereas in other layers (panel d in Fig. 3), there were numerous VGLUT1-ir synapses but no gliosis (Fig. 6C). In conclusion, the reduced number of VGLUT1-ir puncta observed at the light microscope level, was correlated with gliosis, neuronal loss and a decrease in the number of asymmetrical synapses. Therefore, VGLUT1-ir appears to be a very reliable marker to study alterations of excitatory circuits in the human cerebral cortex.

\section{DISCUSSION}

There are three main findings that we can draw from this study. Firstly, we found that the distribution of VGLUT1-ir in the human neocortex is virtually identical to that found in the rat brain, both at the levels of light and electron microscopy. Secondly, VGLUT1-ir is found exclusively at asymmetrical (excitatory) synapses in the human neocortex. Thirdly, the alterations observed in the pattern of 


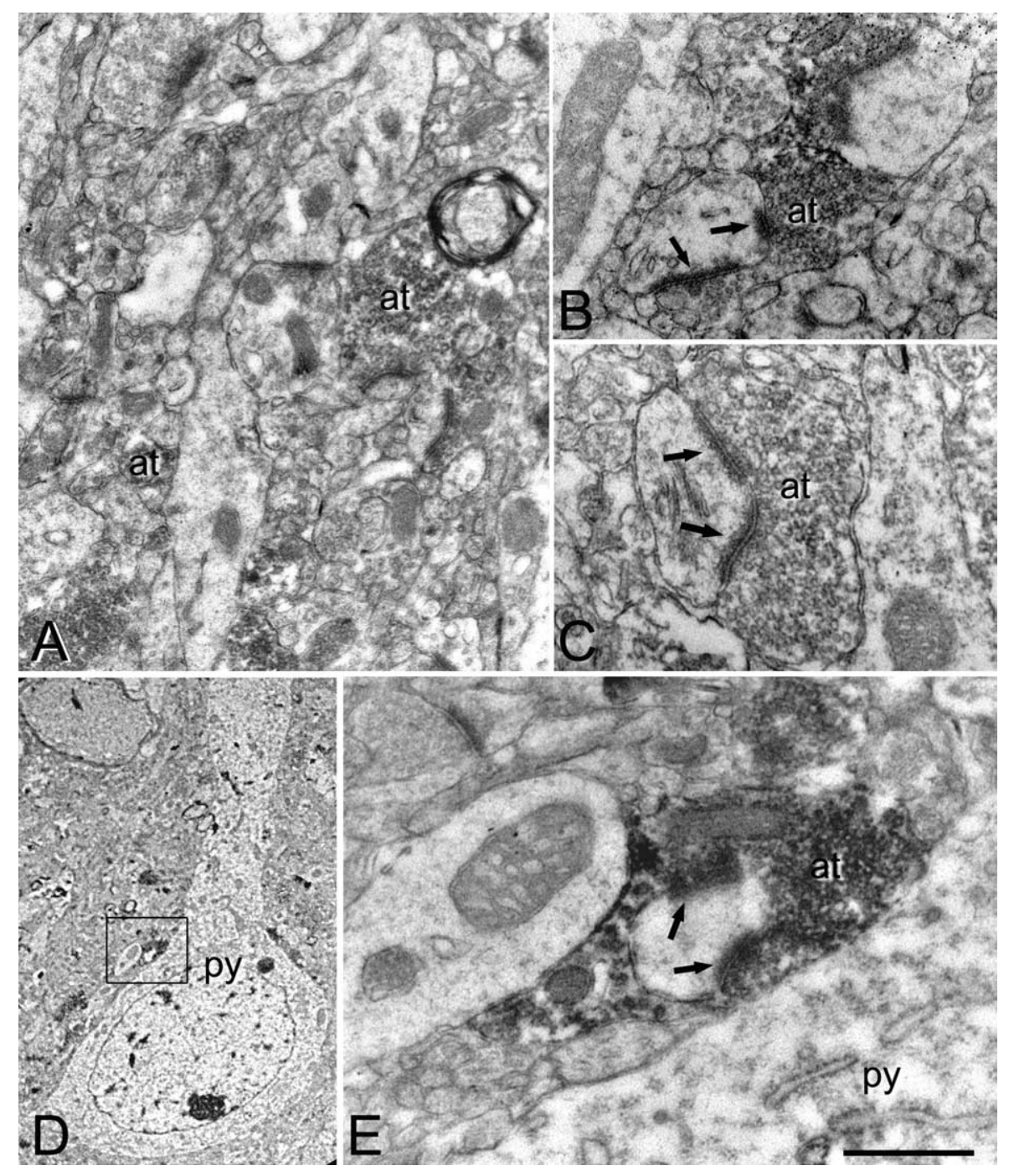

Fig. 5. Electron micrographs illustrating the ultrastructural localization of VGLUT1-ir in the normal human neocortex. (A) Normal appearance of the neuropil from layer III showing some VGLUT1-ir axon terminals (at). (B, C) Higher magnification of VGLUT1-ir at forming asymmetrical synapses (arrows) with dendritic spines. (D) Pyramidal cell body (py) surrounded by perisomatic VGLUT1-ir at. (E) Higher magnification of the boxed area in D, to show a VGLUT1-ir at adjacent to the pyramidal cell membrane (py) forming a perforated asymmetrical synapse with a dendritic profile (arrows). Scale bar $=0.8 \mu \mathrm{m}$ (shown in E) in A, $0.5 \mu \mathrm{m}$ in B, $0.4 \mu \mathrm{m}$ in C, $5.56 \mu \mathrm{m}$ in D and $0.5 \mu \mathrm{m}$ in $\mathrm{E}$.

VGLUT1 immunostaining in the peritumoral neocortex at the light microscope level coincide perfectly with gliosis in the resected tissue, as well as with the decreases in the number of asymmetrical synapses detected by electron microscopy.

In the normal human neocortex, the pattern of immunostaining was very similar to that found in the rat neocortex (reviewed in Kaneko and Fujiyama, 2002; Hisano, 2003). In all layers, punctate structures with a regular distribution were labeled. Moreover, at the ultrastructural level, these VGLUT1-ir profiles exclusively established asymmetrical synapses, as occurs in the rat brain (Bellocchio et al., 1998; Fujiyama et al., 2001; Fremeau et al., 2001; Herzog et al., 2001; Kaneko et al., 2002; AlonsoNanclares et al., 2004). Thus, immunocytochemistry for VGLUT1 appears to be a reliable tool to study a large proportion of the glutamatergic synapses in the human cerebral cortex.

The study of peritumoral neocortical tissue revealed a variable decrease in VGLUT1-ir puncta at the light microscope level, which affected one or several layers. The distribution of these puncta varied between the different cortical regions, as well as between the patients analyzed. It is known that the vast majority of excitatory neurons in the neocortex express VGLUT1 (Kaneko and Fujiyama, 2002; Hisano, 2003; Fremeau et al., 2004a). Furthermore, recent studies have shown that excitatory neurotransmission is greatly reduced in mice when the VGLUT1 gene is knocked out (Fremeau et al., 2004b; Wojcik et al., 2004). Thus, the decrease of VGLUT1-ir in the peritumoral cortex probably reflects the alterations that develop in glutamatergic synaptic circuits. The reduction of VGLUT1-ir in 


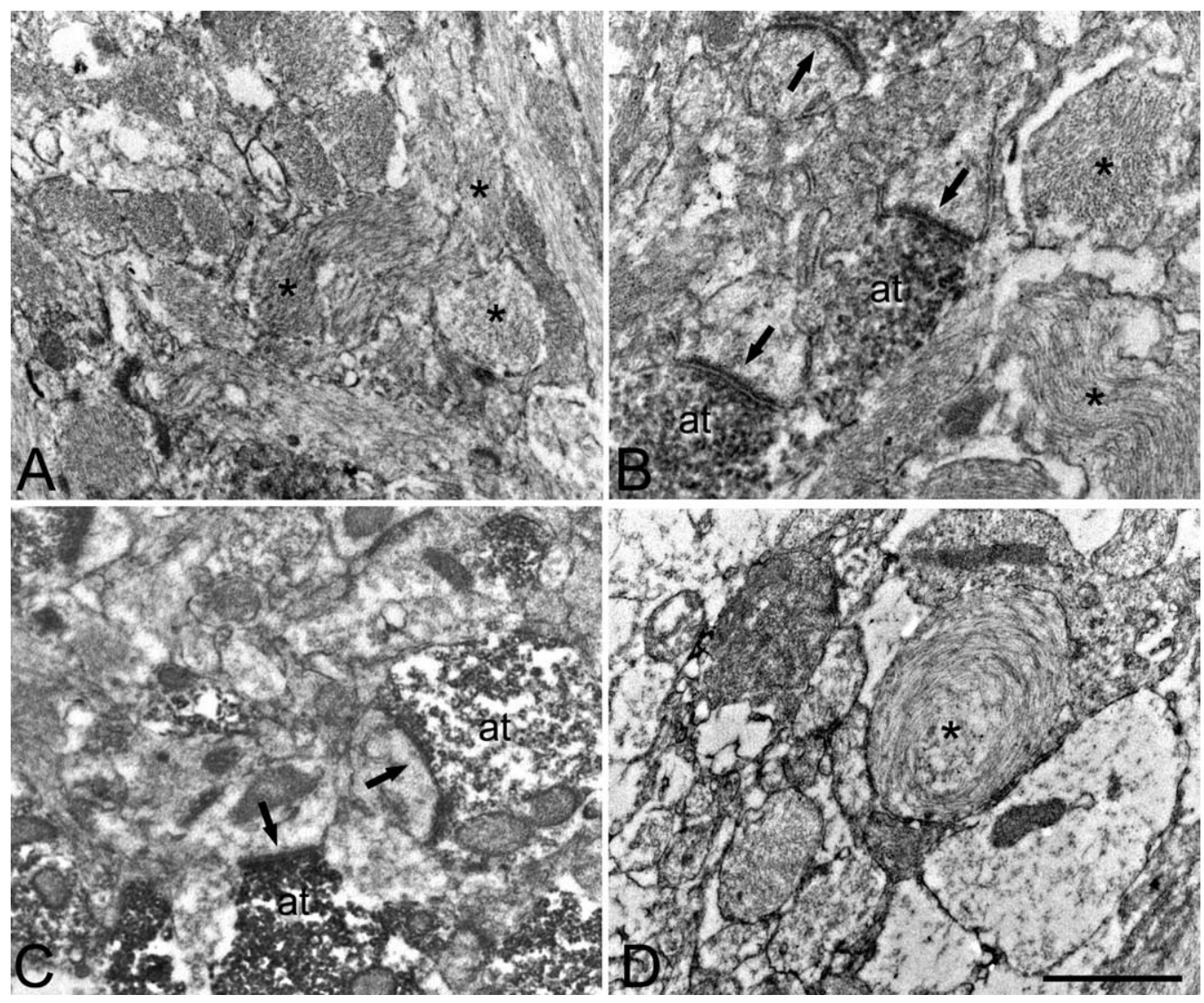

Fig. 6. Electron micrographs illustrating the alterations in the peritumoral neocortex. (A, B) Electron micrographs of layers I (A) and III (B) from patient H66 (see Fig. 2). The neuropil from layer I (A) show numerous glial processes (some of them indicated by asterisks) and a lack of synaptic contacts. In layer III (B), the neuropil shows intense gliosis (asterisks) but relatively numerous, VGLUT1-ir, normal looking axon terminals (at) forming asymmetrical synapses (arrows). (C, D) Electron micrographs from patient H214. In C, the neuropil from layer III is shown, corresponding to panel d in Fig. 3. The VGLUT1-ir at have a normal appearance, establishing asymmetrical synapses (arrows). (D) Micrograph taken from a region corresponding to panel e in Fig. 3 to illustrate the altered neuropil with numerous glial processes (asterisks) and no VGLUT1-ir at. Scale bar $=1.3 \mu \mathrm{m}$ (shown in D) in A, C and D, and $0.5 \mu \mathrm{m}$ in $\mathrm{B}$.

regions of the peritumoral neocortex could be explained by a decrease in the amount of VGLUT1 protein in the axon terminals to below the threshold levels for immunocytochemical detection. However, the decrease in VGLUT1-ir was perfectly matched with the loss of VGLUT1-ir axon terminals at the electron microscope level, and was parallel by a reduction in asymmetrical synapses. Furthermore, in regions showing severe gliosis, VGLUT1-ir axon terminals with a normal appearance were observed but there were no unlabeled axon terminals adjacent to the labeled ones. Thus, the reduction of VGLUT1-ir in the peritumoral cortex did not appear to be due to a decrease in the protein levels in axon terminals induced by the tumoral lesion, but rather to the actual loss of axon terminals. Pyramidal neurons are the most abundant type of cortical neuron and the main source of asymmetrical synapses (White, 1989; DeFelipe and Fariñas, 1992). Since in the peritumoral neocortex there was a clear reduction in the number of neurons in the same regions in which the VGLUT1-ir was diminished, it is very likely that the loss of excitatory axon terminals was due to the loss of the local pyramidal cell arbor resulting from the death of these cells.
In a previous study of human epileptic neocortex not associated to tumors, certain cortical regions that show neuronal loss but not gliosis had more excitatory synapses than adjacent normal regions. This was probably a consequence of the sprouting of pyramidal cell axonal arbors located near to the lesioned areas (Marco and DeFelipe, 1997). It was suggested that this increase in the number of excitatory synapses might be responsible for the hyperexcitability in these regions. However, in peritumoral cortex showing severe gliosis, both neuronal loss and a decrease in the number of synapses was observed (see also Marco et al., 1997). This indicates that the axonal arbor of pyramidal cells located around the lesion has not invaded the regions where gliosis develops, possibly due to the gliosis impeding axonal growth (reviewed in Fawcett and Asher, 1999). Therefore, different alterations of cortical circuits may occur in the human epileptic neocortex associated with neuronal loss, which seems to depend on the glial reaction.

Since the epileptic patients examined in this study responded well to the surgery, it seems likely that the resected cortex contains the epileptogenic region. That is, 
altered cortical circuits that contributed to the hyperexcitability were removed in this resected tissue. However, the remarkable reduction in excitatory synapses in the peritumoral regions where VGLUT1-ir was diminished are probably not involved in epileptogenicity. Moreover, despite the numerous neurons and intense gliosis in these peritumoral regions, the absence of synapses indicated that these neurons were disconnected, making it unlikely that they contribute to epileptiform activity. Therefore, it is possible epileptogenesis arises in more distant normal looking regions, as occurs in cortical lesions associated with dysplasia characterized by similar neuronal loss and gliosis (Alonso-Nanclares et al., 2005). Further studies of the peritumoral cortex will be necessary to determine which alterations of cortical circuits are involved in the generation, maintenance and propagation of epilepsy.

To conclude, VGLUT1-ir puncta represent axon terminals forming asymmetrical synapses and there is a perfect correlation between the decrease of VGLUT1-ir and a decrease in the number of asymmetrical synapses. These characteristics indicate that VGLUT1 immunohistochemistry is a reliable and simple tool to identify alterations in the glutamatergic system, and in the extent and progress of lesions associated with the tumoral pathology.

Acknowledgments-We thank R. F. Senso for technical assistance and J. A. Maldonado for assistance with the electron microscope. This work was supported by the Spanish Ministry of Education and Science (grants PM99-0105, BFI2003-02745, and a research fellowship awarded to L. A.-N., FP2000-4989) and from the 'Comunidad de Madrid' (08.5/0027/2001).

\section{REFERENCES}

Aihara $\mathrm{Y}$, Mashima $\mathrm{H}$, Onda $\mathrm{H}$, Hisano $\mathrm{S}$, Kasuya $\mathrm{H}$, Hori T, Yamada S, Tomura H, Yamada Y, Inoue I, Kojima I, Takeda J (2000) Molecular cloning of a novel brain-type $\mathrm{Na}(+)$-dependent inorganic phosphate cotransporter. J Neurochem 74:2622-2625.

Ajmone-Marsan C, O'Connor M (1973) Electrocorticography. In: Handbook of electroencephalography and clinical neurophysiology, Vol. 10 (Ajmone-Marsan C, ed), pp 3-49. Amsterdam: Elsevier.

Alonso-Nanclares L, Minelli A, Melone M, Edwards RH, DeFelipe J, Conti $F$ (2004) Perisomatic glutamatergic axon terminals: a novel feature of cortical synaptology revealed by vesicular glutamate transporter 1 immunostaining. Neuroscience 123:547-556.

Alonso-Nanclares L, Garbelli R, Sola RG, Pastor J, Tassi L, Spreafico R, DeFelipe J (2005) Microanatomy of the dysplastic neocortex from epileptic patients. Brain 128:158-173.

Avanzini G, de Curtis M, Spreafico R (1992) Physiological properties of GABAergic thalamic reticular neurons studied in vitro: relevance to thalamocortical synchronizing mechanisms. Epilepsy Res Suppl 8:117-124.

Bellocchio EE, Hu H, Pohorille A, Chan J, Pickel VM, Edwards RH (1998) The localization of the brain-specific inorganic phosphate transporter suggests a specific presynaptic role in glutamatergic transmission. J Neurosci 18:8648-8659.

Bellocchio EE, Reimer RJ, Fremeau RT Jr, Edwards RH (2000) Uptake of glutamate into synaptic vesicles by an inorganic phosphate transporter. Science 289:957-960.

Ben Ari Y, Represa A (1990) Brief seizure episodes induce long-term potentiation and mossy fibre sprouting in the hippocampus. Trends Neurosci 13:312-318.
Bernard C, Cossart R, Hirsch JC, Esclapez M, Ben-Ari Y (2000) What is GABAergic inhibition? How is it modified in epilepsy? Epilepsia 41(Suppl 6):S90-S95.

Blümcke I, Lobach M, Wolf HK, Wiestler OD (1999) Evidence for developmental precursor lesions in epilepsy-associated glioneuronal tumors. Microsc Res Tech 46:53-58.

Cascino GD (1990) Epilepsy and brain tumors: implications for treatment. Epilepsia 31(Suppl 3):S37-S44.

Chapman AG (1998) Glutamate receptors in epilepsy. Prog Brain Res 116:371-383.

Chatrian GE, Quesney LF (1999). Intraoperative electrocorticography. In: Epilepsy, the comprehensive text book (Engel J Jr, Pedley TA, eds), pp 1749-1765. Philadelphia: Lippincott, Williams \& Wilkins.

Colonnier M (1968) Synaptic patterns on different cell types in the different laminae of the cat visual cortex. An electron microscope study. Brain Res 9:268-287.

Colonnier M (1981) The electron microscopic analysis of the neuronal organization of the cerebral cortex. In: Organization of the cerebral cortex (Schmitt FO, Worden FG, Adelman G, Dennis SG, eds), pp 125-152. Cambridge: MIT Press.

DeFelipe J, Fariñas I (1992) The pyramidal neuron of the cerebral cortex: morphological and chemical characteristics of the synaptic inputs. Prog Neurobiol 39:563-607.

DeFelipe J, Fairén A (1993) A simple and reliable method for correlative light and electron microscopic studies. J Histochem Cytochem 41:769-772.

DeFelipe J (1999) Chandelier cells and epilepsy. Brain 122:18071822.

Demarque M, Villeneuve N, Manent JB, Becq H, Represa A, Ben Ari $Y$, Aniksztejn $L$ (2004) Glutamate transporters prevent the generation of seizures in the developing rat neocortex. J Neurosci 24:3289-3294.

Engel JJ (1987) Outcome with respect to epileptic seizures. In: Surgical treatment of epilepsies (Engel JJ, ed), pp 553-571. New York: Raven Press.

Fawcett JW, Asher RA (1999) The glial scar and central nervous system repair. Brain Res Bull 49:377-391.

Fremeau RT Jr, Troyer MD, Pahner I, Nygaard GO, Tran CH, Reimer RJ, Bellocchio EE, Fortin D, Storm-Mathisen J, Edwards RH (2001) The expression of vesicular glutamate transporters defines two classes of excitatory synapse. Neuron 31:247-260.

Fremeau RT Jr, Burman J, Qureshi T, Tran CH, Proctor J, Johnson J, Zhang $H$, Sulzer D, Copenhagen DR, Storm-Mathisen J, Reimer RJ, Chaudhry FA, Edwards RH (2002) The identification of vesicular glutamate transporter 3 suggests novel modes of signaling by glutamate. Proc Natl Acad Sci U S A 99:14488-14493.

Fremeau RT Jr, Voglmaier S, Seal RP, Edwards RH (2004) VGLUTs define subsets of excitatory neurons and suggest novel roles for glutamate. Trends Neurosci 27:98-103.

Fremeau RT Jr, Kam K, Qureshi T, Johnson J, Copenhagen DR, Storm-Mathisen J, Chaudhry FA, Nicoll RA, Edwards RH (2004) Vesicular glutamate transporters 1 and 2 target to functionally distinct synaptic release sites. Science 304:1815-1819.

Fujiyama F, Furuta T, Kaneko T (2001) Immunocytochemical localization of candidates for vesicular glutamate transporters in the rat cerebral cortex. J Comp Neurol 435:379-387.

Gras C, Herzog E, Bellenchi GC, Bernard V, Ravassard P, Pohl M, Gasnier B, Giros B, El Mestikawy S (2002) A third vesicular glutamate transporter expressed by cholinergic and serotoninergic neurons. J Neurosci 22:5442-5451.

Gray EG (1959) Electron microscopy of synaptic contacts on dendrite spines of the cerebral cortex. Nature 183:1592-1593.

Helsinki Declaration (1991) BMJ 302:1194.

Herzog E, Bellenchi GC, Gras C, Bernard V, Ravassard P, Bedet C, Gasnier B, Giros B, El Mestikawy S (2001) The existence of a second vesicular glutamate transporter specifies subpopulations of glutamatergic neurons. J Neurosci 21:RC181. 
Hisano S (2003) Vesicular glutamate transporters in the brain. Anat Sci Int 78:191-204.

Houser CR, Harris AB, Vaughn JE (1986) Time course of the reduction of GABA terminals in a model of focal epilepsy: a glutamic acid decarboxylase immunocytochemical study. Brain Res 383:129145.

Kaneko T, Fujiyama F (2002) Complementary distribution of vesicular glutamate transporters in the central nervous system. Neurosci Res 42:243-250.

Kaneko T, Fujiyama F, Hioki H (2002) Immunohistochemical localization of candidates for vesicular glutamate transporters in the rat brain. J Comp Neurol 444:39-62.

Kleihues P, Cavenee WK (2000) Pathology and genetics. Tumors of the nervous system. Lyon: IARC Press.

Marco P, Sola RG, Ramón y Cajal S, DeFelipe J (1997) Loss of inhibitory synapses on the soma and axon initial segment of pyramidal cells in human epileptic peritumoural neocortex: implications for epilepsy. Brain Res Bull 44:47-66.

Marco P, DeFelipe J (1997) Altered synaptic circuitry in the human temporal neocortex removed from epileptic patients. Exp Brain Res 114:1-10.

McNamara JO (1994) Cellular and molecular basis of epilepsy. J Neurosci 14:3413-3425.

Mullen RJ, Buck CR, Smith AM (1992) NeuN, a neuronal specific nuclear protein in vertebrates. Development 116:201-211.

Ni B, Rosteck PR Jr, Nadi NS, Paul SM (1994) Cloning and expression of a cDNA encoding a brain-specific $\mathrm{Na}(+)$-dependent inorganic phosphate cotransporter. Proc Natl Acad Sci U S A 91:5607-5611.

Ni B, Wu X, Yan GM, Wang J, Paul SM (1995) Regional expression and cellular localization of the $\mathrm{Na}(+)$-dependent inorganic phosphate cotransporter of rat brain. J Neurosci 15:5789-5799.

Ni B, Du Y, Wu X, DeHoff BS, Rosteck PR Jr, Paul SM (1996) Molecular cloning, expression, and chromosomal localization of a human brain-specific $\mathrm{Na}(+)$-dependent inorganic phosphate cotransporter. J Neurochem 66:2227-2238.

Peachey LD (1958) Thin sections. I. A study of section thickness and physical distortion produced during microtomy. J Biophys Biochem Cytol 4:233-242.

Peters A (1987) Synaptic specificity in the cerebral cortex. In: Synaptic function (Edelman GM, Gall WE, Cowan WM, eds), pp 373-397. New York: John Wiley.

Peters A, Palay SL, Webster HD (1991) The fine structure of the nervous system. Neurons and their supporting cells. New York: Oxford University Press.
Peters A, Palay SL (1996) The morphology of synapses. J Neurocytol 25:687-700.

Plate KH, Wieser HG, Yasargil MG, Wiestler OD (1993) Neuropathological findings in 224 patients with temporal lobe epilepsy. Acta Neuropathol (Berl) 86:433-438.

Ribak CE, Joubran C, Kesslak JP, Bakay RA (1989) A selective decrease in the number of GABAergic somata occurs in pre-seizing monkeys with alumina gel granuloma. Epilepsy Res 4:126-138.

Sarnat HB, Nochlin D, Born DE (1998) Neuronal nuclear antigen (NeuN): a marker of neuronal maturation in early human fetal nervous system. Brain Dev 20:88-94.

Schwartzkroin PA (1994) Cellular electrophysiology of human epilepsy. Epilepsy Res 17:185-192.

Silva AV, Sanabria ER, Cavalheiro EA, Spreafico R (2002) Alterations of the neocortical GABAergic system in the pilocarpine model of temporal lobe epilepsy: neuronal damage and immunocytochemical changes in chronic epileptic rats. Brain Res Bull 58:417-421.

Sloviter RS (1991) Permanently altered hippocampal structure, excitability, and inhibition after experimental status epilepticus in the rat: the "dormant basket cell" hypothesis and its possible relevance to temporal lobe epilepsy. Hippocampus 1:41-66.

Spreafico R, Mennini T, Danober L, Cagnotto A, Regondi MC, Miari A, De Blas A, Vergnes M, Avanzini G (1993) GABAA receptor impairment in the genetic absence epilepsy rats from Strasbourg (GAERS): an immunocytochemical and receptor binding autoradiographic study. Epilepsy Res 15:229-238.

Takamori S, Malherbe P, Broger C, Jahn R (2002) Molecular cloning and functional characterization of human vesicular glutamate transporter 3. EMBO Rep 3:798-803.

Varoqui H, Schafer MK, Zhu H, Weihe E, Erickson JD (2002) Identification of the differentiation-associated $\mathrm{Na}+/ \mathrm{PI}$ transporter as a novel vesicular glutamate transporter expressed in a distinct set of glutamatergic synapses. J Neurosci 22:142-155.

White EL (1989) Cortical circuits: synaptic organization of the cerebral cortex. Boston: Birkhäuser Boston.

Wojcik SM, Rhee JS, Herzog E, Sigler A, Jahn R, Takamori S, Brose $\mathrm{N}$, Rosenmund C (2004) An essential role for vesicular glutamate transporter 1 (VGLUT1) in postnatal development and control of quantal size. Proc Natl Acad Sci U S A 101:7158-7163.

Wolf HK, Buslei R, Schmidt-Kastner R, Schmidt-Kastner PK, Pietsch T, Wiestler OD, Bluhmke I (1996) NeuN: a useful neuronal marker for diagnostic histopathology. J Histochem Cytochem 44:11671171. 